\title{
Erratum to: Evidence of statistical epistasis between DISC1, CIT and NDEL1 impacting risk for schizophrenia: biological validation with functional neuroimaging
}

\author{
Kristin K. Nicodemus • Joseph H. Callicott $\cdot$ Rachel G. Higier • Augustin Luna - Devon C. Nixon • \\ Barbara K. Lipska - Radhakrishna Vakkalanka - Ina Giegling • Dan Rujescu • David St. Clair • \\ Pierandrea Muglia $\cdot$ Yin Yao Shugart $\cdot$ Daniel R. Weinberger
}

Published online: 2 February 2010

(C) Springer-Verlag 2010

\section{Erratum to: Hum Genet}

DOI 10.1007/s00439-009-0782-y

Authors regret that Figs. 2 and 3 labeling in the online published article, color indication for graph bars "Non-risk" and "Risk" were inadvertently swapped. The corrected figures are given below.

The online version of the original article can be found under doi:10.1007/s00439-009-0782-y.

K. K. Nicodemus · J. H. Callicott - R. G. Higier - A. Luna

D. C. Nixon · B. K. Lipska · R. Vakkalanka ·

D. R. Weinberger $(\square)$

Genes, Cognition and Psychosis Program,

Intramural Research Program, National Institute

of Mental Health, National Institutes of Health,

Room 4S-235, 10 Center Drive,

Bethesda, MD 20892, USA

e-mail: weinberd@mail.nih.gov

\section{K. K. Nicodemus}

Wellcome Trust Centre for Human Genetics,

University of Oxford, Roosevelt Road,

Oxford OX3 7BN, UK

e-mail: kristin.nicodemus@well.ox.ac.uk

\section{K. K. Nicodemus}

Department of Clinical Pharmacology,

Old Road Campus Research Building,

University of Oxford, Old Road Campus,

Off Roosevelt Drive, Headington,

Oxford OX3 7DQ, UK
I. Giegling · D. Rujescu

Section of Molecular and Clinical Neurobiology,

Department of Psychiatry, Ludwig Maximilians University,

Nussbaumstrasse 7, 80336 Munich, Germany

D. St. Clair

Institute of Medical Sciences, University of Aberdeen,

Foresterhill, Aberdeen AB25 2ZD, UK

P. Muglia

GlaxoSmithKline, Via Fleming 4, Verona 37135, Italy

Y. Y. Shugart

Department of Epidemiology,

Johns Hopkins Bloomberg School of Public Health,

615 N Wolfe St, Baltimore, MD 21205, USA

\section{Y. Y. Shugart}

Genomic Research Branch, Division of Neuroscience Center, National Institute of Mental Health, National Institutes of Health, Room 7191, 6001 Executive Blvd,

Bethesda, MD 20892, USA 

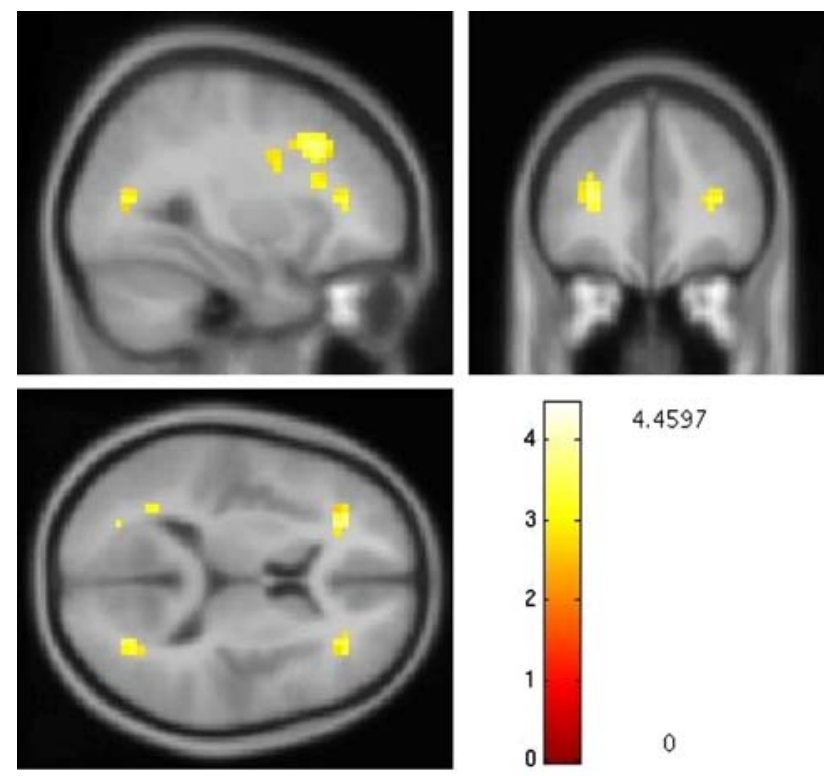

4.4597

0

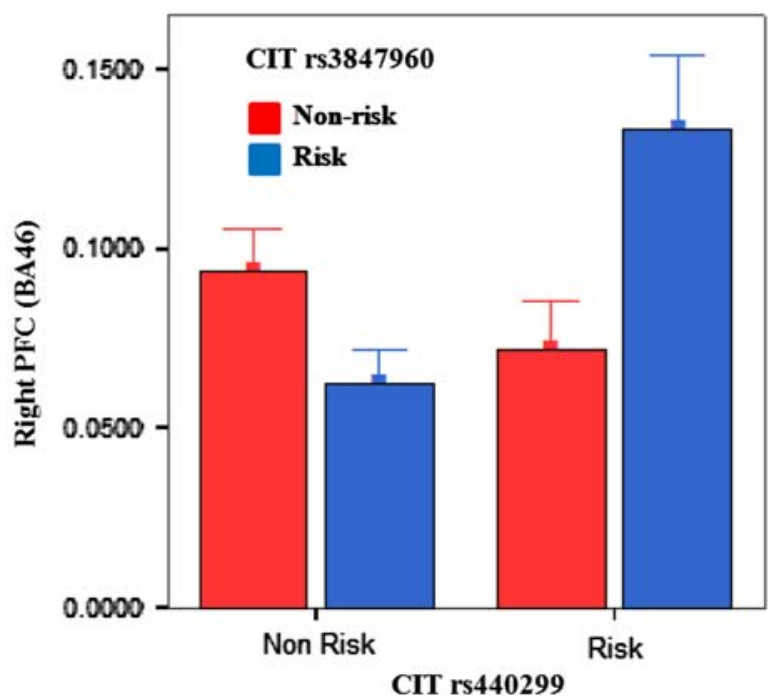

Fig. 2 CIT intragenic neuroimaging epistasis. CIT rs 3847960 by CIT rs440299 interaction in normal subjects studied with BOLD fMRI during the $\mathrm{N}$ back working memory task. Figure at the top shows loci (in yellow) within brain in which significant interaction is found $(p<0.05$ corrected) for all voxels within prefrontal cortical region of interest. Image at bottom shows relative degree of activation of DLPFC region showing imaging interaction based on genotypes at these two SNPs [fMRI signal extracted from maximum voxel and run as ANOVA in SPSS yielded $F(1,256)=9.960, p=0.002]$. The combination of both risk associated genotypes is disproportionally inefficient, i.e., have greatest activation without any difference in performance. Error bars represent 1 standard error of the mean. Frontal lobe (sub-gyral) mean activation extracted from $10 \mathrm{~mm}$ sphere at (30 37 8) Talairach
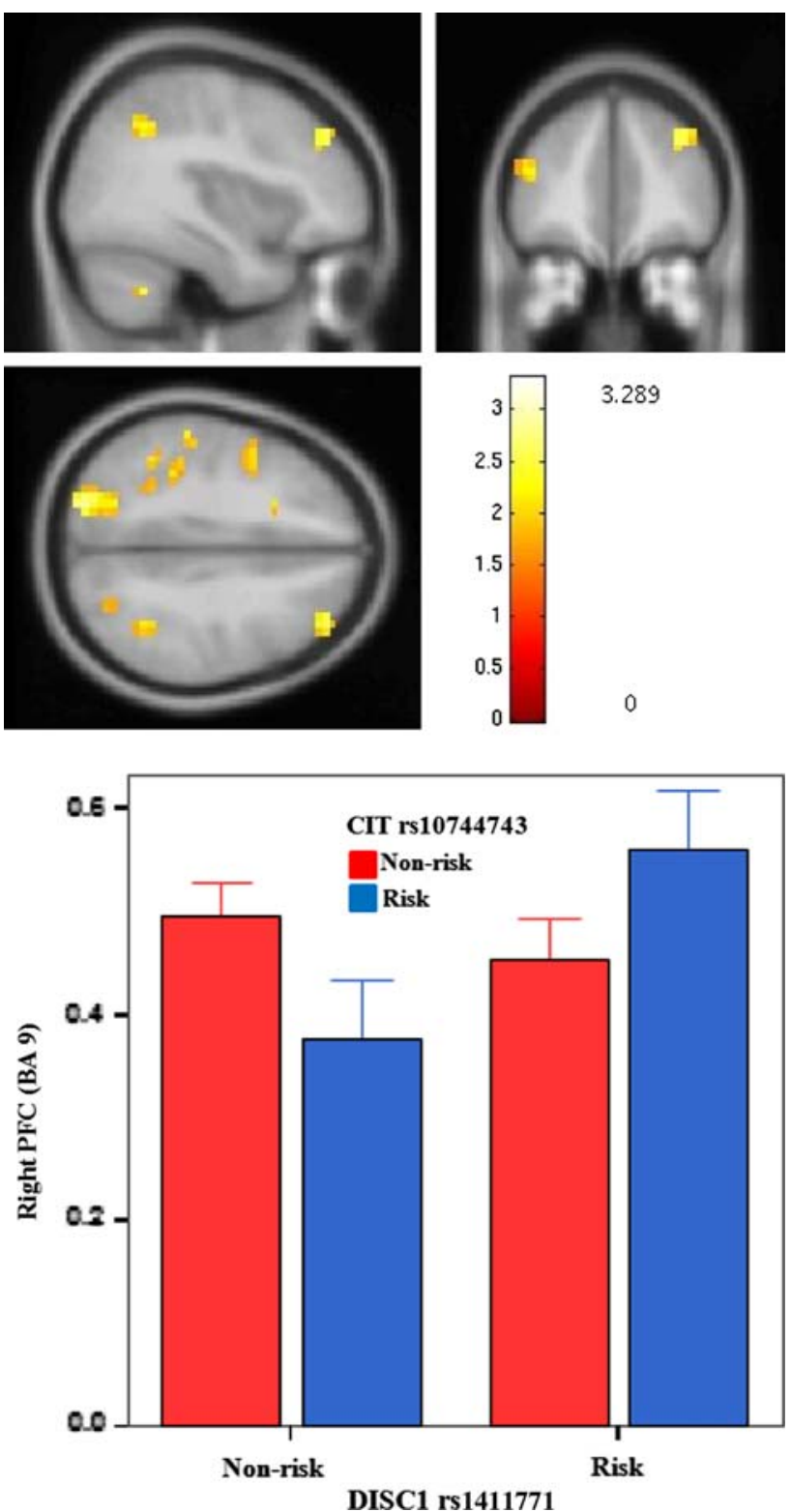

Fig. 3 DISC1 by CIT neuroimaging epistasis. DISC1 rs1411771 by CIT rs10744743 interaction in 217 normal subjects studied with BOLD fMRI during the $N$-back working memory task. The crosssectional brain images at top figure show loci (in yellow) within brain showing a significant inefficiency effect associated with DISC1 risk $\times$ CIT risk SNPs $(p<0.05$ small volume correction SVC). The graph at bottom compares a measure of fMRI activation during this task for each genotype combination extracted from right PFC yielding a significant epistatic interaction [fMRI signal extracted from maximum voxel and run as ANOVA in SPSS yielded $F(1,213)=5.3 p<0.05]$ 